\title{
Efeito da suplementação com berberina nas dislipidemias em humanos: Uma revisão
}

\section{sistemática}

\author{
Effect of supplementation with berberine on dyslipidemias in humans: A systematic review \\ Efecto de la suplementación con berberina sobre las dislipidemias en humanos: Una revisión \\ sistemática
}

Recebido: 06/04/2021 | Revisado: 13/04/2021 | Aceito: 15/04/2021 | Publicado: 29/04/2021

Débora Martins Cavalheiro

ORCID: https://orcid.org/0000-0001-7230-2313

Universidade Federal do Piauí, Brasil

E-mail: deboracavalheiro@ufpi.edu.br

Keylla de Araújo Silva

ORCID: https://orcid.org/0000-0002-8957-091X

Universidade Federal do Piauí, Brasil

E-mail: keyllaaraujo96@gmail.com

Bruna Emanuele Pereira Cardoso

ORCID: https://orcid.org/0000-0002-3863-1276

Universidade Federal do Piauí, Brasil

E-mail: brunaemanuelec@hotmail.com

Thaline Milany da Silva Dias

ORCID: https://orcid.org/0000-0003-1734-4312 Universidade Federal do Piauí, Brasil

E-mail: thatadias77@gmail.com

Alana Rafaela da Silva Moura

ORCID: https://orcid.org/0000-0002-4089-8623 Universidade Federal do Piauí, Brasil

E-mail: alanarafaelasmoura@gmail.com

Stéfany Rodrigues de Sousa Melo ORCID: https://orcid.org/0000-0001-5308-3522 Universidade Federal do Piauí, Brasil E-mail: stefany.rsm@gmail.com

Maiara Jaianne Bezerra Leal Rios ORCID: https://orcid.org/0000-0003-2349-7235 Universidade Federal do Piauí, Brasil E-mail: maiararios2014\#gmail.com

Jennifer Beatriz Silva Morais

ORCID: https://orcid.org/0000-0002-9055-7851 Universidade Federal do Piauí, Brasil

E-mail: jenniferbeatriz.morais@gmail.com

\begin{abstract}
Resumo
Objetivo: descrever em uma revisão sistemática, os desfechos de ensaios clínicos relativos à suplementação com berberina e dislipidemias em humanos. Metodologia: A pesquisa dos artigos foi realizada nas bases de dados Pubmed, ScienceDirect, Cochrane e Lilacs. A estratégia PICO (patient, intervention, comparison and outcomes) foi utilizada para a definição da pergunta norteadora desta revisão. Foram utilizados os termos "Coptis chinensis OR Berberine" AND "Dyslipidemia OR Dyslipoproteinemias" para o levantamento bibliográfico. Foram incluídos nessa revisão sistemática ensaios clínicos randomizado, duplo cego, placebo controlados, publicados em inglês, sem limite de ano de publicação, que avaliaram o efeito da suplementação com berberina sobre a influência nos perfis lipídicos em humanos com dislipidemia, sem restrição de gênero, idade e etnia. Resultados: Após o processo de triagem, remoção de artigos duplicados e análises dos critérios de inclusão, 10 artigos foram identificados como elegíveis para esta revisão sistemática. Discussão: a maioria dos ensaios clínicos incluídos encontraram redução dos níveis de colesterol total, triglicérides e LDL. Os desfechos de todos os estudos foram similares, indicando que a berberina e seus respectivos compostos bioativos, apresentam potencial no tratamento de dislipidemias. Conclusão: Os resultados desta revisão sistemática fornecem evidencias dos benefícios da suplementação da berberina sobre o perfil lipídico, colesterol total, triglicérides e a redução dos níveis de LDL em indivíduos dislipidêmicos. No entanto, novos estudos de intervenção são necessários para extrapolar os resultados desse estudo em diferentes populações, bem como padronizar doses do extrato da berberina para suplementação.
\end{abstract}

Palavras-chave: Berberina; Coptis Chinenses; Dislipidemia. 


\begin{abstract}
Objective: to describe in a systematic review, the outcomes of clinical trials related to supplementation with berberine and dyslipidemia in humans. Methodology: The search for the articles was carried out in the Pubmed, ScienceDirect, Cochrane and Lilacs databases. The PICO strategy (patient, intervention, comparison and outcomes) was used to define the guiding question for this review. The terms "Coptis chinensis OR Berberine" AND "Dyslipidemia OR Dyslipoproteinemias" were used for the bibliographical survey. Included in this systematic review were randomized, double-blind, placebo controlled clinical trials, published in English, with no year limit of publication, which evaluated the effect of supplementation with berberine on the influence on lipid profiles in humans with dyslipidemia, without gender restriction, age and ethnicity. Results: After the screening process, removal of duplicate articles and analysis of the inclusion criteria, 10 articles were identified as eligible for this systematic review. Discussion: most of the included clinical trials found a reduction in the levels of total cholesterol, triglycerides and LDL. The outcomes of all studies were similar, indicating that berberine and its respective bioactive compounds have potential in the treatment of dyslipidemia. Conclusion: The results of this systematic review provide evidence of the benefits of berberine supplementation on the lipid profile, total cholesterol, triglycerides and the reduction of LDL levels in dyslipidemic individuals. However, further intervention studies are needed to extrapolate the results of this study in different populations, as well as to standardize doses of berberine extract for supplementation.
\end{abstract}

Keywords: Berberine; Coptis Chinenses; Dislipidemia.

\title{
Resumen
}

Objetivo: describir en una revisión sistemática los resultados de los ensayos clínicos relacionados con la suplementación con berberina y la dislipidemia en humanos. Metodología: La búsqueda de los artículos se realizó en las bases de datos Pubmed, ScienceDirect, Cochrane y Lilacs. Se utilizó la estrategia PICO (paciente, intervención, comparación y resultados) para definir la pregunta guía para esta revisión. Los términos "Coptis chinensis OR Berberine" Y "Dislipidemia OR Dislipoproteinemias" se utilizaron para la encuesta bibliográfica. En esta revisión sistemática se incluyeron ensayos clínicos aleatorizados, doble ciego, controlados con placebo, publicados en inglés, sin límite de año de publicación, que evaluaron el efecto de la suplementación con berberina sobre la influencia en los perfiles lipídicos en humanos con dislipidemia, sin restricción de género., edad y etnia. Resultados: Después del proceso de selección, eliminación de artículos duplicados y análisis de los criterios de inclusión, se identificaron 10 artículos como elegibles para esta revisión sistemática. Discusión: la mayoría de los ensayos clínicos incluidos encontraron una reducción en los niveles de colesterol total, triglicéridos y LDL. Los resultados de todos los estudios fueron similares, lo que indica que la berberina y sus respectivos compuestos bioactivos tienen potencial en el tratamiento de la dislipidemia. Conclusión: Los resultados de esta revisión sistemática proporcionan evidencia de los beneficios de la suplementación con berberina sobre el perfil lipídico, el colesterol total, los triglicéridos y la reducción de los niveles de LDL en individuos dislipidémicos. Sin embargo, se necesitan más estudios de intervención para extrapolar los resultados de este estudio en diferentes poblaciones, así como para estandarizar las dosis de extracto de berberina para la suplementación.

Palabras clave: Berberina; Coptis Chinenses; Dislipidemia.

\section{Introdução}

A dislipidemia é definida por mudanças na concentração de um ou mais lípides/lipoproteínas presentes na corrente sanguínea (triglicérides, colesterol, lipoproteínas de alta e de baixa densidade). Essas mudanças no perfil lipídico estão diretamente relacionadas ao desenvolvimento de aterosclerose (Xavier, et al., 2013).

As dislipidemias são um fator de risco para as doenças cardiovasculares, essa patologia é responsável por cerca de 4,4 milhões de mortes por ano em todo o mundo (Li, et al., 2014). O colesterol, apesar de ser o principal foco de ação neste tipo de patologias, é uma importante molécula e de caráter essencial a algumas funções vitais do nosso organismo. A manutenção da integridade celular, a produção de hormonas esteroides e ácidos biliares são algumas das funções atribuídas a esta molécula (Zhang, Temel, Martel, 2014).

Dados mostram que uma diminuição de $1.0 \mathrm{mmol} / \mathrm{l}$ nas lipoproteínas de baixa densidade (LDL) pode resultar numa redução de cerca de $9 \%$ na taxa de mortalidade geral e de aproximadamente de $25 \%$ nos episódios vasculares, mesmo entre os doentes de baixo risco (Li, et al., 2014).

O tratamento mais utilizado atualmente a fim de diminuir o colesterol LDL é estatina. Porém, nem sempre esse tratamento é bem tolerado (Pierro, 2016). Foram informados vários efeitos colaterais decorrentes da terapia com estatinas, como elevação da transaminase, maior risco para diabetes mellitus e, em alguns casos, correlações com disfunções renais e 
neurológicas (Wilkinson, Laffin, Davidson, 2014).

Embora a medicina tenha avançado e evoluído a partir do século XX, as plantas ainda têm grande contribuição para a manutenção da saúde e melhora de patologias em países em desenvolvimento (Souza \& Felfili, 2006). Evidências científicas indicam o crescimento da utilização da fitoterapia como prática integrativa no mundo todo. No Brasil, a prática representa uma utilização do vasto potencial de sua flora medicinal e agrega recurso terapêutico de baixo custo (Santos, et al., 2011).

Atualmente, outros tratamentos estão disponíveis, como berberina. A berberina é extraída de várias plantas da família Berberidaceae, nomeadamente Berberis aristata (cúrcuma), Berberis vulgaris (bérberis) e Berberis aquifolium (a uva Oregon). Têm se destacado, através de estudos, potenciais efeitos da berberina com base em mecanismos farmacológicos relacionados ao metabolismo, inclusive, no tratamento de hipercolesterolemia (Vuddanda, Chakraborty, Singh, 2010).

Dessa forma, considerando a importância das dislipidemias e os desfechos associados a ela, a exemplo das doenças cardiovasculares que configuram como um grave problema de saúde pública, a relevância da ingestão de fitoterápicos, a exemplo da berberina no controle do perfil lipídico, bem como a inconsistência de dados na literatura sobre essas variáveis, torna-se evidente a realização de estudos de revisão que possam esclarecer e melhor fundamentar a relação entre essas variáveis.

Portanto, o objetivo desse trabalho foi descrever em uma revisão sistemática, os desfechos de ensaios clínicos relativos à suplementação com berberina e dislipidemias em humanos.

\section{Metodologia}

\section{Fontes de Dados e Seleção de Estudos}

O presente estudo consiste em uma revisão sistemática da literatura (Pereira, et al., 2018). A pesquisa dos artigos foi realizada por duas autoras de forma independente. As pesquisas realizadas pelas referidas autoras foram comparadas, sendo verificada equivalência na busca e seleção dos artigos. A pesquisa dos artigos foi realizada nas bases de dados Pubmed, ScienceDirect, Cochrane e Lilacs. A estratégia PICO (patient, intervention, comparison and outcomes) foi utilizada para a definição da pergunta norteadora dessa revisão (Tabela 1). Foram utilizados os termos "Coptis chinensis OR Berberine" AND "Dyslipidemia OR Dyslipoproteinemias" para o levantamento bibliográfico.

Tabela 1. PICO - critérios de inclusão de estudos.

\begin{tabular}{lr} 
Parâmetro & Critério \\
\hline Participantes/População & Pacientes com dislipidemia. \\
Intervencão & Suplementação com berberina. \\
Comparação & Com placebo ou controle sem comparação. \\
Resultados & Diminuição dos níveis de colesterol total, LDL e triglicérides. \\
Tipo de estudo & Ensaio clínico
\end{tabular}

Fonte: Dados dos autores.

Foram incluídos nessa revisão sistemática ensaios clínicos randomizado, duplo cego, placebo controlados, publicados em inglês, sem limite de ano de publicação, que avaliaram o efeito da suplementação com berberina sobre a influência nos 
perfis lipídicos em humanos com dislipidemia, sem restrição de gênero, idade e etnia. Não foram incluídos nessa revisão relatos de caso, estudos transversais, caso-controle, artigos de revisão e pesquisas realizadas em animais. Os detalhes da seleção dos estudos são apresentados na Figura 1, a seguir:

Figura 1. Seleção de estudos para a revisão sistemática.

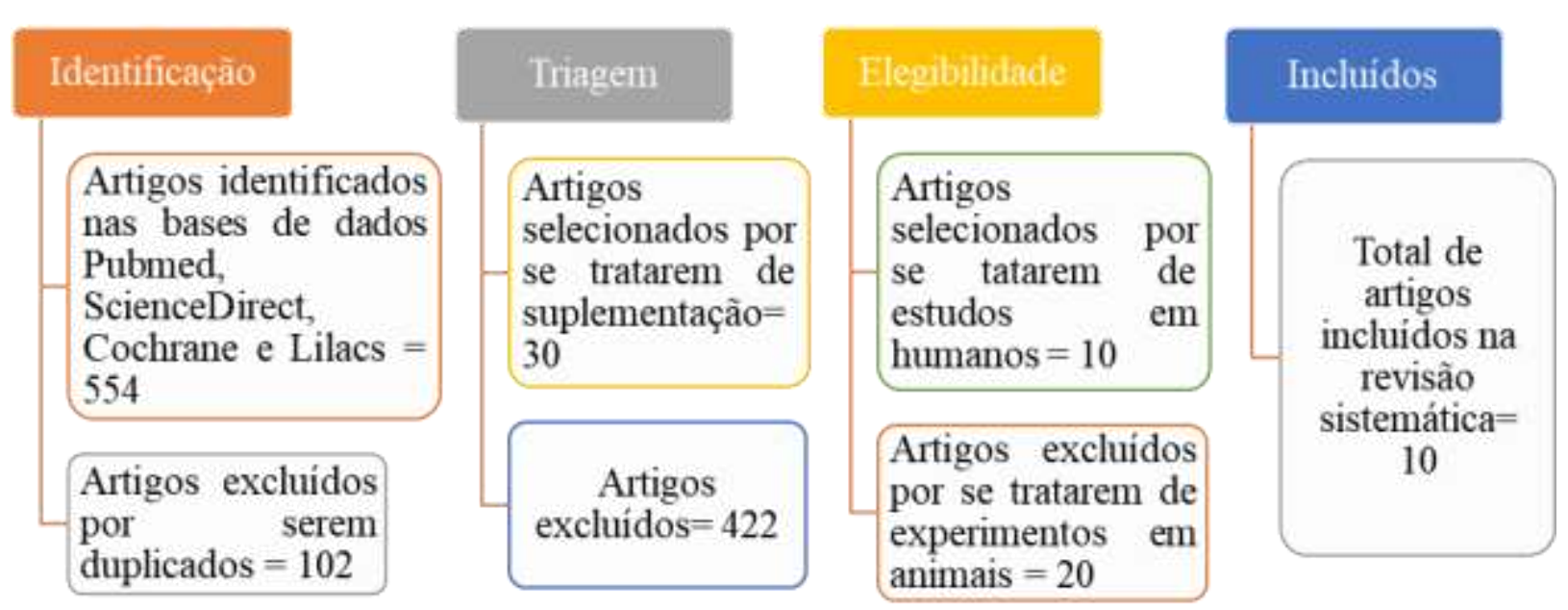

Fonte: Dados dos autores.

Informações relevantes serão coletadas dos artigos dessa revisão, incluindo autores, ano de publicação, local de realização da pesquisa, tamanho da amostra, sexo e idade da população estudada, dose e duração da suplementação, grupo controle ou placebo, métodos de avaliação dos parâmetros do perfil lipídico associados à berberina.

\section{Avaliação do Risco de Vieses}

As recomendações do Preferred Reporting Items for Systematic Reviews and Meta-Analyses (PRISMA) foram seguidas para realização dessa revisão. A Cochrane collaboration's tool foi utilizada para avaliar o risco de viés dos ensaios clínicos incluídos nesse estudo. 
Figura 2. Avaliação dos riscos de viés.

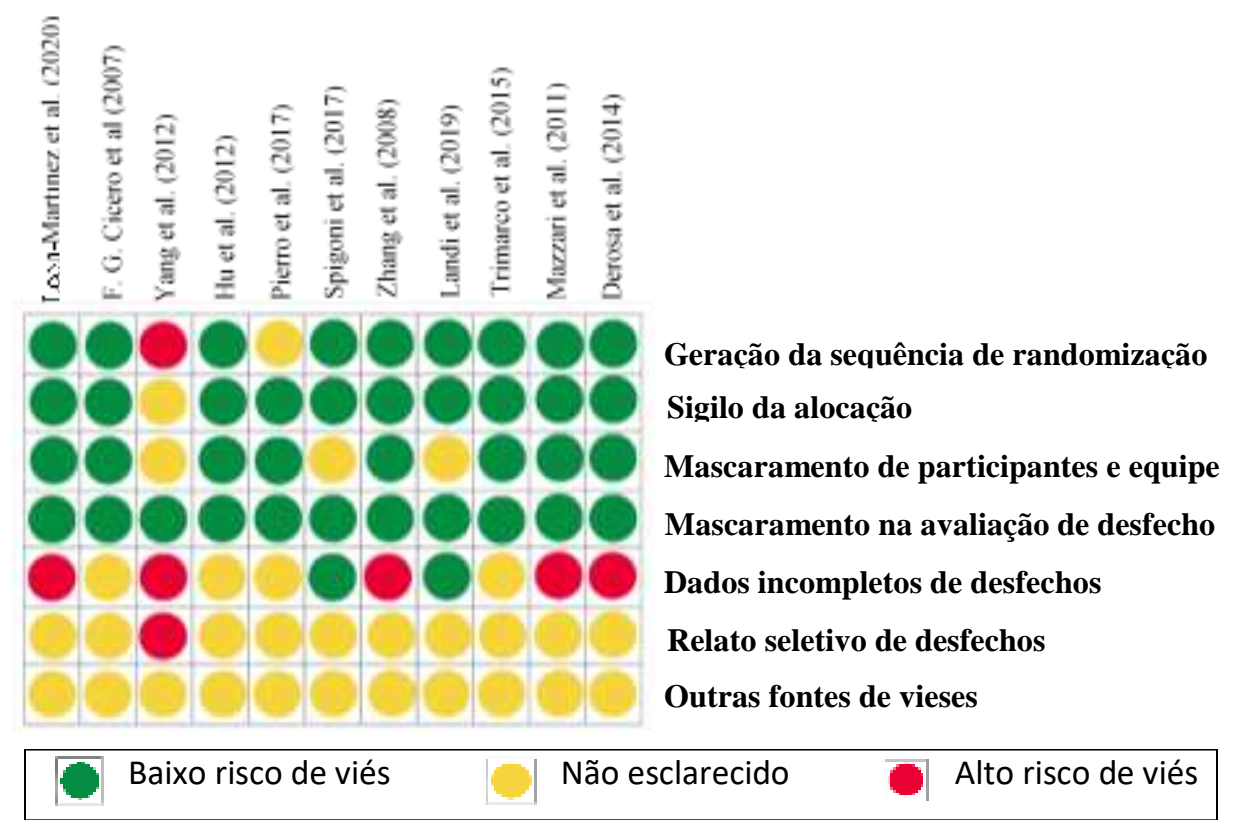

Fonte: Dados dos autores.

\section{Resultados}

\section{Artigos Selecionados}

Um total de 554 artigos foram identificados por meio de pesquisa nas bases de dados PubMed ( $\mathrm{n}=165)$, ScienceDirect $(n=389)$, Cochrane $(n=0)$ e Lilacs $(n=0)$. Após o processo de triagem, remoção de artigos duplicados e análises dos critérios de inclusão, 10 artigos foram identificados como elegíveis para esta revisão sistemática. A Tabela 2 mostra as características dos artigos incluídos. 
Tabela 2. Características dos artigos incluídos.

\begin{tabular}{|c|c|c|c|c|c|c|c|c|c|}
\hline \multicolumn{10}{|c|}{ Participantes (n) } \\
\hline Autores & $\begin{array}{c}\text { Ano da } \\
\text { publicação }\end{array}$ & País & $\begin{array}{l}\text { Supleme } \\
\text { nto }\end{array}$ & Placebo & $\begin{array}{c}\text { Gêner } \\
0\end{array}$ & $\begin{array}{l}\text { Idade } \\
\text { (anos) }\end{array}$ & Dose & Duração & Principal desfecho \\
\hline $\begin{array}{l}\text { Leon-Martinez } \\
\text { et al. }\end{array}$ & 2020 & $\begin{array}{c}\text { Méxic } \\
0\end{array}$ & 24 & 12 & $\mathrm{~F} / \mathrm{M}$ & $30-60$ & BBR $(500 \mathrm{mg})$ oral & 12 semanas & $\begin{array}{c}\downarrow \mathrm{CT}, \mathrm{LDL}-\mathrm{Ce} \\
\text { VLDL }\end{array}$ \\
\hline Landi et al. & 2019 & Itália & 23 & 24 & $\mathrm{~F} / \mathrm{M}$ & $18-75$ & $\begin{array}{c}\text { Comprimido } \\
\text { (500mg BBR + } \\
\text { outros })\end{array}$ & 6 semanas & $\begin{array}{l}\downarrow \mathrm{CT}, \mathrm{LDL} \cdot \mathrm{Ce} \\
\text { triglicérides }\end{array}$ \\
\hline Pierro et al. & 2017 & Itália & 31 & 28 & $\mathrm{~F} / \mathrm{M}$ & $\geq 18$ & Berberol (500mg) & 24 semanas & $\downarrow$ HbAlc $\downarrow \mathrm{CT}$ \\
\hline Spigoni et al. & 2017 & Itália & 30 & 9 & $\mathrm{~F} / \mathrm{M}$ & 52 & BBR (500mg) & 12 semanas & $\begin{array}{l}\downarrow \text { LDL-C } \downarrow \text { LDLL } \downarrow \\
\text { Apolipoproteina }\end{array}$ \\
\hline Trimarco et al. & 2015 & Itália & 23 & - & $\mathrm{F} / \mathrm{M}$ & $18-70$ & $\begin{array}{c}\text { Comprimido } \\
\text { (500mg BBR + } \\
\text { outros })\end{array}$ & 8 semanas & $\downarrow$ LDL-C \\
\hline Derosa et al. & 2015 & Itália & 81 & 82 & $\mathrm{~F} / \mathrm{M}$ & $\geq 18$ & Berberol (588mg) & 24 semanas & $\downarrow$ Glicose \\
\hline Hu et al. & 2012 & EUA & 8 & - & $\mathrm{F} / \mathrm{M}$ & 40 & BBR (500mg) & 12 semanas & $\downarrow$ CT e TG \\
\hline Yang et al. & 2012 & $\begin{array}{c}\text { Chin } \\
\text { a }\end{array}$ & 9 & - & $\mathrm{F} / \mathrm{M}$ & $22-47$ & BBR $(10 \mu g)$ & 12 semanas & $\begin{array}{c}\downarrow \text { leptina, adiponectina, } \\
\text { HOMA-IR }\end{array}$ \\
\hline Marazzi et al. & 2011 & Itália & 40 & 40 & $\mathrm{~F} / \mathrm{M}$ & $>75$ & $\begin{array}{c}\text { Comprimido } \\
\text { (500mg BBR + } \\
\text { outros })\end{array}$ & 48 semanas & $\downarrow \mathrm{CT} \downarrow$ LDL-C \\
\hline Zhang et al. & 2008 & $\begin{array}{l}\text { Chin } \\
\text { a }\end{array}$ & 116 & - & $\mathrm{F} / \mathrm{M}$ & $25-70$ & $\begin{array}{l}\text { Comprimido de } \\
\operatorname{BBR}(0.25 \mathrm{~g})\end{array}$ & 12 semanas & $\downarrow \mathrm{LDL} \downarrow \mathrm{TG}$ \\
\hline Cicero et al & 2007 & Itália & 20 & 29 & F/M & $57-63$ & $\begin{array}{l}\text { Comprimido } \\
\text { (500mg BBR) }\end{array}$ & 4 semanas & $\downarrow$ CT e TG \\
\hline
\end{tabular}

Fonte: Dados dos autores.

A partir da avaliação dos 10 estudos incluídos nesta revisão, observou-se que os ensaios clínicos foram realizados com adultos, de ambos os sexos, em quatro países diferentes, sendo que as doses de suplementação de berberina variaram de 200mg a $588 \mathrm{mg} /$ dia. A duração das intervenções foi entre 4 semanas a 12 meses, nas formas de rizoma em comprimidos e cápsulas de berberina.

\section{Qualidade metodológica dos estudos incluídos}

A avaliação da qualidade metodológica dos estudos incluídos nesta revisão corresponde ao que está apresentada na Tabela 2. Os estudos satisfazem a maioria dos critérios estabelecidos para avaliar a qualidade metodológica, segundo a Cochrane collaboration's tool.

\section{Efeito da suplementação com berberina sobre o controle da dislipidemia}

Sobre os efeitos da suplementação de berberina na dislipidemia, todos os estudos que avaliaram esse parâmetro encontraram redução do perfil lipídico com a suplementação da berberina.

O estudo de $\mathrm{Hu}$ et al. (2012) que envolveu sujeitos obesos durante 12 semanas, foi realizado para avaliar a eficácia e segurança da berberina. Assim, foram sujeitos a um tratamento de $500 \mathrm{mg}$ de berberina, três vezes por dia. No final do estudo, não só reduziram os níveis de colesterol total, quando comparados com os valores pré-tratamento (12,2\%), como também foi notória a redução nos níveis de triglicerídeos $(23 \%)$. Este estudo avaliou também a influência na redução de marcadores 
inflamatórios cardiovasculares, no entanto não se notaram alterações significativas a nível da proteína C-reativa (redução de $12,3 \%)$.

Zhang et al. (2008) realizou sua pesquisa com o objetivo de avaliar a eficácia e segurança no tratamento de doentes com diabetes tipo 2 e com dislipidemia sem tratamento prévio, sujeitando os voluntários a um tratamento com berberina $(0,5$ mg duas vezes por dia) ou placebo. O estudo teve a duração de 3 meses e os resultados demonstraram que as concentrações de colesterol total, triglicerídeos e LDL no grupo tratado reduziram significativamente quando comparadas com as alterações no grupo placebo. Este estudo mostrou assim, que após três meses de tratamento, se conseguiu uma redução de $18 \%$ nos níveis séricos de colesterol, 35,9\% nos níveis de TG e de $21 \%$ nos níveis de LDL. Podemos afirmar assim, que a berberina, mostrouse mais eficaz na redução dos níveis de TG do que colesterol, mostrando um efeito similar aos Fibratos.

No estudo realizado por Leon-martinez et al. (2020), a avaliação clínica, perfil lipídico, níveis de glicose e acido úrico foram medidos antes e após a intervenção farmacológica. Os resultados mostraram que a berberina reduziu os níveis de colesterol total e a berberina em conjunto com o bezafibrato diminuíram significativamente as concentrações de TG, TC, LDLC e VLDL e, portanto, remitindo o diagnóstico de dislipidemia mista em $90 \%$ dos pacientes.

Acerca do colesterol total, seis dos onze estudos incluídos demonstraram redução desse parâmetro, sendo que todos esses encontraram redução do colesterol total com a suplementação de berberina. No que diz respeito aos níveis de triglicérides quatro artigos incluídos nessa revisão verificaram melhora nos valores de triglicérides com a suplementação de berberina e sete dos onze estudos mostraram a redução de LDL com a suplementação de berberina.

\section{Discussão}

Esta revisão sistemática foi realizada na perspectiva de investigar o efeito da suplementação com berberina no tratamento de dislipidemias. Com relação ao efeito da intervenção sobre o perfil lipídico, a maioria dos ensaios clínicos incluídos encontraram redução dos níveis de colesterol total, triglicérides e LDL.

Um ponto importante a ser destacado nesta discussão, diz respeito às discordâncias nas doses de suplementação, bem como na forma de suplementação da berberina, biodisponibilidade e o tempo de suplementação, que podem ter influenciado alguns parâmetros utilizados para avaliar os níveis de colesterol. A maioria dos estudos foram realizados na Itália, os outros se dividiram em México, China e Estados Unidos.

No entanto, apesar das divergências metodológicas encontradas, os desfechos de todos os estudos foram similares, indicando que a berberina e seus respectivos compostos bioativos, apresentam potencial no tratamento de dislipidemias. Mesmo em doses menores (200mg) até doses elevadas (588g), a berberina parece ter efeito benéfico no controle dislipidêmico, sem efeitos de toxicidade.

A berberina mostrou eficácia na redução lipídica comparativamente às estatinas. Nos estudos in vitro, utilizando linhas hepatocelulares de carcinoma humano, mostrou um aumento da expressão do gene do recetor das LDL, a um nível póstranscricional, conseguido através da estabilização do mRNA, por meio da ativação da via extracelular das cinases reguladas por sinal. Clarificando este mecanismo, a estabilização do mRNA do recetor das LDL é mediada pela via de sinalização ERK, através de interações de sequências cis-reguladoras da região 3' não traduzida no mRNA do receptor das LDL. Este mecanismo difere das estatinas na medida em que é totalmente independente dos níveis de colesterol e não possui qualquer efeito na atividade da HMG-CoA redutase (Affuso, et al., 2010).

A berberina interfere no metabolismo lipídico através da regulação da função dos receptores hepáticos de LDL (Wei, et al., 2012). Dong et al. (2013) analisaram a ação da berberina no perfil lipídico e verificaram uma redução significativa dos níveis de LDL-C e de TG com esta substância.

Os efeitos da berberina no metabolismo dos lípidos estão sendo estudados e sabe-se que a berberina diminui os níveis 
dos lípidos através do aumento de expressão do receptor hepático do colesterol associado às LDL (LDLR) pela regulação do sinal de uma cinase extracelular (ERK) e ainda por aumentar a atividade transcripcional do promotor LDL-R pela via da cinase do fator de transcrição c-Jun (JNK) (Hu \& Davies, 2010).

Para além do aumento da expressão do LDLR, a berberina ativa a cinase de adenosina monofosfato (AMPK), enquanto que bloqueia a via da cinase das proteínas ativadoras da mitogénese/ERK (MAPK/ERK), resultando na inibição da síntese lipídica (Cicero \& Ertek, 2009).

A berberina atua no metabolismo dos lípidos inibindo a adipogénese durante a diferenciação dos pré-adipócitos. Este fármaco vai atuar sobre o colesterol total e triglicerídeo e ainda promove uma diminuição da ingestão de alimentos o que conduz a uma diminuição do peso corporal. Outra vantagem deste composto é o fato de não apresentar toxicidade para os animais, sendo segura a sua utilização (Hu \& Davies, 2010).

A grande maioria dos fitoterápicos utilizados atualmente por automedicação ou por prescrição não tem o seu perfil toxico bem conhecido (Veiga-Junior, 2008). Entretanto, a utilização inadequada de um produto, mesmo de baixa toxicidade, pode induzir problemas graves desde que existam outros fatores de risco tais como contraindicações ou uso concomitante de outros medicamentos (Amorim, et al., 2007).

Uma limitação que deve ser mencionada, diz respeito à possibilidade de artigos relevantes não terem sido incluídos porque foram publicados em língua diferente de inglês ou em fontes não indexadas nas bases de dados eletrônicas acessadas.

\section{Conclusão}

Os resultados dessa revisão sistemática fornecem evidencias dos benefícios da suplementação da berberina sobre o perfil lipídico, colesterol total, triglicérides e a redução dos níveis de LDL em indivíduos dislipidêmicos. No entanto, novos estudos de intervenção são necessários para extrapolar os resultados desse estudo em diferentes populações, bem como padronizar doses do extrato da berberina para suplementação.

Nesse sentido, faz-se importante estudos futuros que esclareçam ainda mais os mecanismos pelos quais a suplementação com berberina se relacionam com marcadores lipídicos em indivíduos dislipidêmicos.

\section{Referências}

Amorim, M. F. D., Diniz, M. F. F. M., Araújo, M. S. T., Pita, J. C. L. R.; Dantas, J. G., Ramalho, J. A., Xavier, A. L., Palomaro, T. V., \& Júnior, N. L. B. (2007).

The controvertible role of kava (Piper methysticum G. Foster) an anxiolytic herb, on toxic hepatitis. Revista Brasileira de Farmacognosia, 17(3), 448-454.

Affuso, F., Mercurio, V., Fazio, V., \& Fazio, S. (2010). Cardiovascular and metabolic effects of Berberine. World Journal of Cardiology, $2(4), 71-77$.

Cicero, A., Rovati, L., \& Setnikar, I. (2007). Eulipidemic Effects of Berberine Administered Alone or in Combination with Other Natural Cholesterollowering Agents. Arzneimittelforschung, 57(01), 26-30.

Cicero, A., \& Ertek, S. (2009). Berberine: metabolic and cardiovascular effects in preclinical and clinical trials. Nutrition and Dietary Supplements, 1, 1-10.

Derosa, G., Romano, D., D'angelo, A., \& Maffioli, P. (2015). Berberis aristata/Silybum marianum fixed combination (Berberol®) effects on lipid profile in dyslipidemic patients intolerant to statins at high dosages: a randomized, placebo-controlled, clinical trial. Phytomedicine, 22(2), $231-237$.

Dong, H., Zhao, Y., Zhao, L., \& Lu, F. (2013). The Effects of Berberine on Blood Lipids: a systemic review and meta-analysis of randomized controlled trials. Planta Medica, 79(6), 437-446.

Hu, Y., \& Davies, G. E. (2010). Berberine inhibits adipogenesis in high-fat diet-induced obesity mice. Fitoterapia, 81(5), 358-366.

Hu, Y., Ehli, E. A., Kittelsrud, J., Ronan, P. J., Munger, K., Downey, T., Bohlen, K., Callahan, L., Munson, V., Jahnke, M., Marshall, L. L., Nelson, K., Huizenga, P., Hansen, R., Soundy, T. J., \& Davies, G. E. (2012). Lipid-lowering effect of berberine in human subjects and rats. Phytomedicine, 19(10), 861867.

Landi, F., Martone, A. M., Salini, S., Zazzara, B., Calvani, R., Marzetti, E., Nesci, A., Giorgio, A., Giupponi, B., \& Santoro, L. (2019). Effects of a New Combination of Medical Food on Endothelial Function and Lipid Profile in Dyslipidemic Subjects: a pilot randomized trial. Biomed Research International, $2019,1-7$. 
León-Martínez, J. M., Martínez-Abundis, E., González-Ortiz, M., \& Pérez-Rubio, K. G. (2020). Effect of Berberine Plus Bezafibrate Administration on the Lipid Profile of Patients with Mixed Dyslipidemia: a pilot clinical trial. Journal of Medicinal Food, 00(0), 1-5.

Marazzi, G., Cacciotti, L., Pelliccia, F., Iaia, L., Volterrani, M., Caminiti, G., Sposato, B., Massaro, R., Grieco, F., \& Rosano, G. (2011). Long-term effects of nutraceuticals (berberine, red yeast rice, policosanol) in elderly hypercholesterolemic patients. Advances in Therapy, 28(12), 1105-1113.

Pereira, A. S., Shitsuka, D. M., Parreira, F. J. \& Shitsuka, R. (2018). Metodologia da pesquisa científica. UFSM. https://repositorio.ufsm.br/bitstream/ha ndle/1/15824/Lic_Computacao_Metodologia-Pesquisa

Pierro F. (2016). Berberina. Topics on Biopharmaceutical Phytotherapy. Cec Editore. Italia.

Pierro, F., Putignano, P., Ferrara, T., Raiola, C., Rapacioli, G., \& Villanova, N. (2017). Retrospective analysis of the effects of a highly standardized mixture of Berberis aristata, Silybum marianum, and monacolins K and KA in patients with dyslipidemia. Clinical Pharmacology: Advances and Applications, 9, 1-7.

Souza, C. D.; \& Felfili, J. M. (2006). Uso de plantas medicinais na região de Alto Paraíso de Goiás, GO, Brasil. Acta Botânica Brasileira, 20(1), 135-142.

Santos, R. L., Guimaraes, G. P., Nobre, M. S. C. \& Portela, A. S. (2011). Análise sobre a fitoterapia como prática integrativa no Sistema Único de Saúde. Revista brasileira de plantas medicinais, 13(4), 486-491.

Spigoni, V., Aldigeri, R., Antonini, M., Micheli, M., Fantuzzi, F., Fratter, A., Pellizzato, M., Derlindati, E., Zavaroni, I., Bonadonna, R. (2017). Effects of a New Nutraceutical Formulation (Berberine, Red Yeast Rice and Chitosan) on Non-HDL Cholesterol Levels in Individuals with Dyslipidemia: results from a randomized, double blind, placebo-controlled study. International Journal of Molecular Sciences, 18(7), 1-15.

Trimarco, V., Izzo, R., Stabile, E., Rozza, F., Santoro, M., Manzi, M. V., Serino, F., Schiattarella, G. G., Esposito, G., \& Trimarco, B. (2015). Effects of a New Combination of Nutraceuticals with Morus alba on Lipid Profile, Insulin Sensitivity and Endotelial Function in Dyslipidemic Subjects. A Cross-Over, Randomized, Double-Blind Trial. High Blood Pressure \& Cardiovascular Prevention, 22(2), 149-154.

Veiga-Junior, V. F. (2008). Estudo do consumo de plantas medicinais na Região Centro-Norte do Estado do Rio de Janeiro: aceitação pelos profissionais de saúde e modo de uso pela população. Revista Brasileira de Farmacognosia, 18(2), 308-313.

Vuddanda, P. R., \& Chakraborty, S., Singh, S. (2010). Berberine: a potential phytochemical with multispectrum therapeutic activities. Expert Opinion On Investigational Drugs, 19(10), 1297-1307.

Wilkinson, M. J., Laffin, L. J., \& Davidson, M. H. (2014). Superando a toxicidade e os efeitos colaterais das terapias hipolipemiantes. Best Practice \& Research Clinical Endocrinology \& Metabolism, 28, 439-452.

Wei, W., Zhao, H., Wang, A., Sui, M., Liang, k., Deng, H., Ma, Y., Zhang, Y., Zhang, H., \& Guan, Y. (2012). A clinical study on the short-term effect of berberine in comparison to metformin on the metabolic characteristics of women with polycystic ovary syndrome. European Journal of Endocrinology, $166(1), 99-105$

Yang, J., Yin, J., Gao, H., Xu, L., Wang, Y., Xu, L., \& Li, M. (2012). Berberine Improves Insulin Sensitivity by Inhibiting Fat Store and Adjusting Adipokines Profile in Human Preadipocytes and Metabolic Syndrome Patients. Evidence-Based. Complementary and Alternative Medicine, $2012,1-9$.

Zhang, H., Temel, R. E., \& Martel, C. (2014). Cholesterol and Lipoprotein Metabolism. Arteriosclerosis, Thrombosis, and Vascular Biology, 34(9), 17911794.

Zhang, Y., Li, X., Zou, D., Liu, W., Yang, J., Zhu, N., Huo, L., Wang, M., Hong, J., Wu, P., Ren, G., \& Ning, G. (2008). Treatment of Type 2Diabetes and Dyslipidemia with the Natural Plant Alkaloid Berberine. The Journal of Clinical Endocrinology \& Metabolism, 93(7), 2559-2565. 